\title{
Evaluation of Recording Methods for User Test Sessions on Mobile Devices
}

\author{
Andrea Abney, Brooke White*, Jeremy Glick*, Andre Bermudez, Paul Breckow, Jason Yow*, \\ Rayna Tillinghast-Trickett, Paul Heath \\ Disney Interactive \\ 1200 Grand Central Ave, Glendale, CA 91201 \\ andrea.abney@disney.com, andre.bermudez@disney.com,paul.breckow@disney.com, rayna.tillinghast- \\ trickett@disney.com, paul.c.heath@disney.com
}

\begin{abstract}
Disney Interactive produces mobile games, applications, and websites. The user research group is responsible for evaluating games and apps through user testing. We have been working on refining our video capture and streaming solutions for user tests on mobile devices.

We designed this experiment to see if any of the recording methods we used were changing player behavior and impacting their gameplay performance. We assessed changes in absolute score for two different games to determine impact to player efficacy. We analyzed observational data and player self-ratings on performance, comfort, awareness, and focus. We evaluated children, young adults, and older adults.

The results across all of the data were consistent and this paper explains the experiment and provides recommendations for mobile recording of user test sessions.
\end{abstract}

\section{Author Keywords}

Mobile user testing; usability testing; mobile recording methods; games user research; usability lab design.

\section{ACM Classification Keywords}

H.5.2. User Interfaces: Evaluation/methodology.

\section{INTRODUCTION}

In recent years, the user research group at Disney Interactive has been asked with growing frequency to conduct studies on mobile games in addition to our console and PC-based testing. Studies run on mobile games now account for a significant portion of all user research at Disney Interactive. The user research group at Disney Interactive has adapted over the past few years to address the evolving needs of the company. This shifted focus to

Permission to make digital or hard copies of part or all of this work for personal or classroom use is granted without fee provided that copies are not made or distributed for profit or commercial advantage and that copies bear this notice and the full citation on the first page. Copyrights for thirdparty components of this work must be honored. For all other uses, contact the Owner/Author(s). Copyright is held by the owner/author(s).

CHI PLAY'14, Oct 19-22 2014, Toronto, ON, Canada

ACM 978-1-4503-3014-5/14/10.

http://dx.doi.org/10.1145/2658537.2658704 the mobile experience inspired further research into updated recording solutions for mobile research.

Mobile games are increasingly using gestural user interfaces that require careful evaluation. The screen's small size and touch-based interface can introduce unique usability challenges, including illegible user interface elements, small or overlapping hit boxes, and instances where players' fingers or hands obscure part of the screen. To be able to evaluate the unique usability challenges on mobile devices, it is important to evaluate interactions with the device hardware in addition to interactions with the game software. The form factor of mobile devices can cause players to hold them in their hands near their bodies. This makes observation challenging, especially for the development teams, who need to be able to observe from outside the test lab.

The Disney Interactive user research group has experimented with variations of three different solutions, all of which have their respective strengths and weaknesses:

1. Direct "over the shoulder" observation with no recording device: This method allows the researcher to observe the player directly interacting with the device as naturally as possible in a test facility with an observer. However, it is ineffective for game development teams, who are unable to observe the gameplay outside of the test facility and video recordings are not an option.

2. Overhead camera observation and recording: Using this method, a camera is suspended over players' hands by either utilizing a document camera or a webcam attached to a stand. The camera simultaneously streams and records images of the players' hands and the gameplay on the screen. Players do not have to hold an additional object, allowing for a potentially more natural play experience. However, the user is still required to keep the mobile device under the camera, resulting in frequent observation challenges resulting from player posture obscuring the camera and players' tilting the mobile screen away from the camera. 
3. Camera sled observation and recording: In this configuration, the mobile device and camera are both attached to a device called a sled, which players hold in their hands. This keeps the camera at a fixed distance between players' hands and the device, but allows players to hold and move the device without interfering with the camera's line of sight.

We used two different mobile recording sleds.

The white plastic sled [6] comes as a flat piece of precut plastic. We had to bend the sled and attach our own webcam. In contrast, the clear plastic sled [7] came as a finished product with an attached document camera. It did not require additional finishing. The clear sled is smaller and lighter than the white sled. It has a smaller camera, making the top portion less intrusive in the players' field of view. We had concerns about the weight, balance, and intrusiveness of the white sled.

Our researchers hypothesized that our recording methods might be negatively impacting player behavior and performance. The teams and our researchers were concerned that these solutions for mobile testing might be impacting participant behavior.

These factors led us to study this issue in an attempt to find the potentially least intrusive observation and streaming method for mobile user testing.

\section{RELATED WORKS}

A 2005 study [2] examined the differences between using an overhead document camera and a wireless mobile recording sled for recording user tests. To test each device, the researchers used the same script and asked participants to complete the same tasks. They conducted the document camera tests in a lab and the mobile sled tests in the field. In the document camera condition, the phone was attached to a tripod placed under the camera, which kept participants from moving the phone around. Though the results showed that there were mainly similarities between the two, there were a few important differences. Participants using the document camera took more time to complete the tasks and gave up more frequently. They were also less satisfied with their experience. The researchers found the greatest number of usability problems in the document camera condition, but this could be due to environmental differences caused by testing the document camera in the lab and the wireless sled in the field.

In 2007, researchers from PayPal [4] recorded usability tests with a mobile device taped to a table to limit its movement and also conducted cognitive walkthrough sessions where participants were allowed to hold the device naturally. They did not find any differences in task completion between the two methods. It's important to note that all results from this study are purely observational. The researchers did not look at any quantitative measures.

Also in 2007, Google researchers [9] outlined the criteria for a mobile observation setup, including infrastructure issues, mobile device-specific issues, and usability study context issues. They pointed out that one of the most important factors is allowing participants to hold the device naturally in order to capture accurate interaction information. The bulk of the paper outlines their iterations on their mobile recording setup. They found the sled's weight and the user's comfort were important to running a successful mobile study with natural interaction. After a few iterations, they developed a lightweight wooden sled.

In a related paper, Carolyn Wei of Google [10] discussed multiple methods for capturing mobile phone usage,
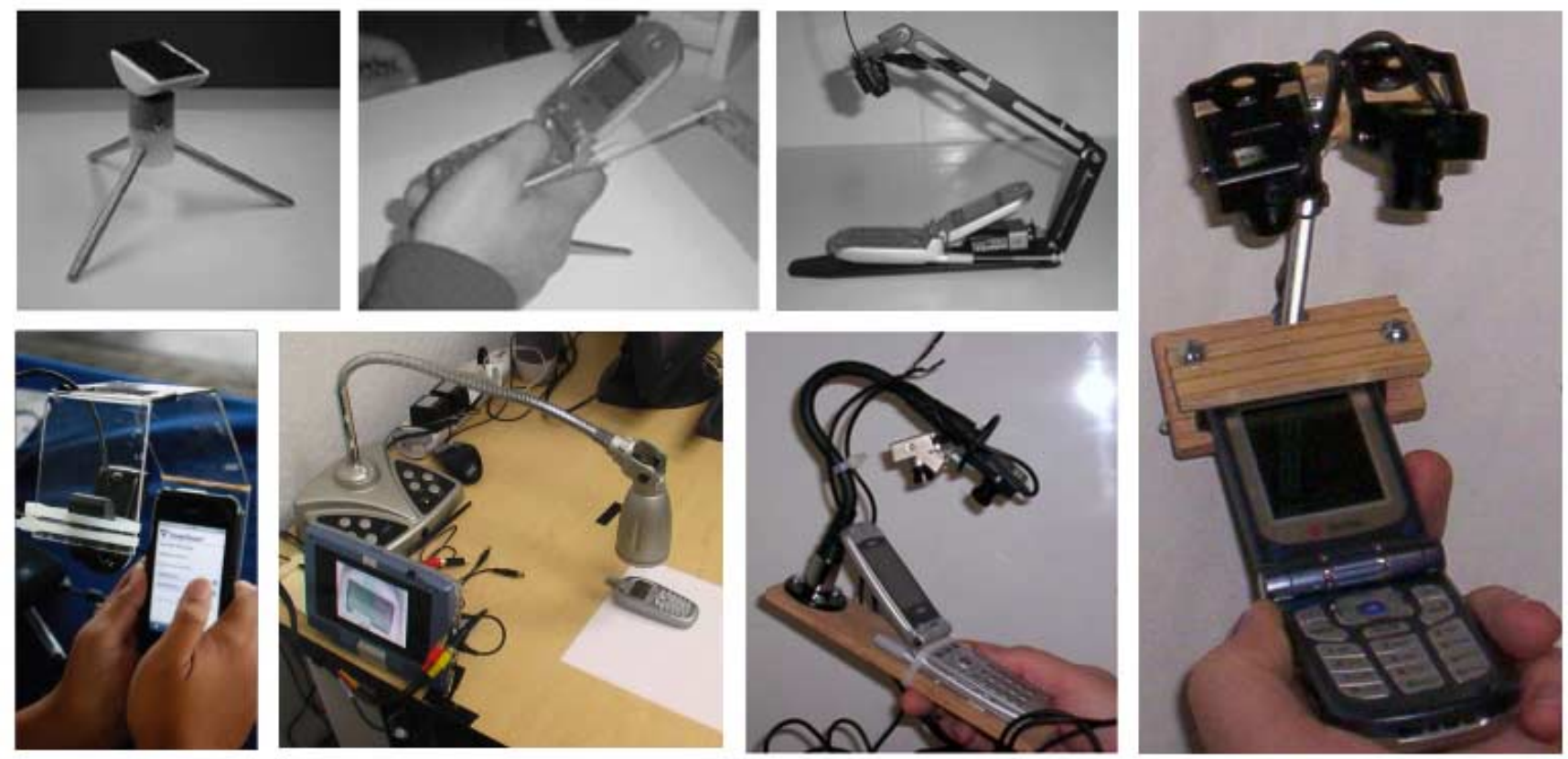

Figure 1. Previous mobile recording devices (clockwise from top left): Tripod [2], phone attached to tripod [2], wireless mobile sled [2], wooden clamp sled [10], wooden base sled [10], document camera over phone [10], clear mobile sled [8] 
including interviews, surveys, diaries, and video recordings of behavior. She observed that video recording behavior with cameras mounted to mobile devices might result in the most accurate data collection. There are some drawbacks, such as wires and obtrusive equipment, which make this method more suited to lab studies rather than field studies.

A more recent study [8] detailed the creation of a mobile sled that is similar to the sleds used in our study. Unlike the previous sleds, this sled was designed to be used with a touchscreen smartphone. When building the sled, the researchers had several requirements: participants had to be able to hold the phone naturally while a webcam recorded the screen, the solution had to use existing equipment as much as possible, and the device had to look professional.

Some researchers have developed more novel approaches to mobile recording, including cameras and equipment built into clothing and accessories [3,5]. We have discussed experimenting with wearable recording equipment in our labs, but we have chosen to focus on refining the sled devices. We may revisit wearable recording devices in the future. Further research in this area could compare wearable devices to handheld sleds.

There is still work to be done in developing solutions for recording on mobile devices. Many other researchers have gone through the process of iterating on recording solutions and no current solution is without its drawbacks. Mobile gaming presents some new challenges and nothing in the literature directly addresses them, creating the need for us to develop our own test of solutions for recording mobile games user research sessions.

\section{METHODS}

To determine whether or not our recording methods were impacting participant behavior and understand the magnitude of any discovered changes, we designed a counterbalanced, within-subjects experiment. We started with a null hypothesis stating that player performance should not be affected by data capture method.

\section{Participants}

Thirty participants residing in and around the Glendale, California area participated in the study. They ranged in age from 8 to 60 . Ten participants were 8 to 12 years old, ten were 18 to 35 years old, and ten were 40 to 60 years old. None of these participants had participated in any prior usability research studies, so none of them had experience with the recording devices used in the study. Participants' gaming experience ranged from casual to hardcore, but all had experience playing games on iOS devices. They were each compensated $\$ 100$.

\section{Materials}

Mobile Devices

The mobile device used in the study was the iPhone $4 \mathrm{~S}$, which weighs 140 grams.

\section{Headphones}

The headphones used were generic, full size headphones with a 90 degree bent headphone jack.

\section{Games}

Participants played two mobile games, Ridiculous Fishing: A Tale of Redemption and Fruit Ninja. We selected these games because they are action-oriented titles with game rounds that are short in length. Ridiculous Fishing was played in portrait orientation while Fruit Ninja was played in landscape orientation. Ridiculous Fishing relies on the use of the iPhone's accelerometer. Fruit Ninja does not use the accelerometer but requires rapid gestural interaction.

For Ridiculous Fishing, the game parameters were $200 \mathrm{~m}$ line, shotgun equipped, basic lure, and "Home Waters" map with no tech equipped. For the Fruit Ninja trials, participants played in the 60 -second arcade mode.

\section{Recording Devices}

- Overhead Camera: A webcam is attached to a plastic stand $40 \mathrm{~cm}$ tall. The webcam is pointed down at the table.

- White Sled: The sled is made of white plastic and is 20 $\mathrm{cm}$ tall. The camera is attached to the top of the sled with a binder clip and rubber bands, which allow the camera to be adjusted forward or backward as needed. The sled and camera weigh 161.59 grams. The phone was attached to this sled with adhesive dots.

- Clear Sled: This sled is made of clear plastic and is 18 $\mathrm{cm}$ tall. The camera is adhered to the top of sled with double-sided tape. The sled features an additional piece that slides on the bottom to support the phone while in landscape orientation. Without the additional piece, the sled and camera weigh 110.56 grams. With the additional piece, they weigh 172.93 grams. Adhesive dots were also used with this sled.

\section{Design}

There were four conditions and players played both games in each condition:
A. No recording device (control)
B. Overhead camera
C. White sled
D. Clear sled

This study used a counterbalanced, within-subjects design. Each participant went through the conditions in a different order to reduce sequencing and order effects.

\section{Procedure}

We set up four stations and ran participants in groups. Three of the stations contained one mobile recording device 

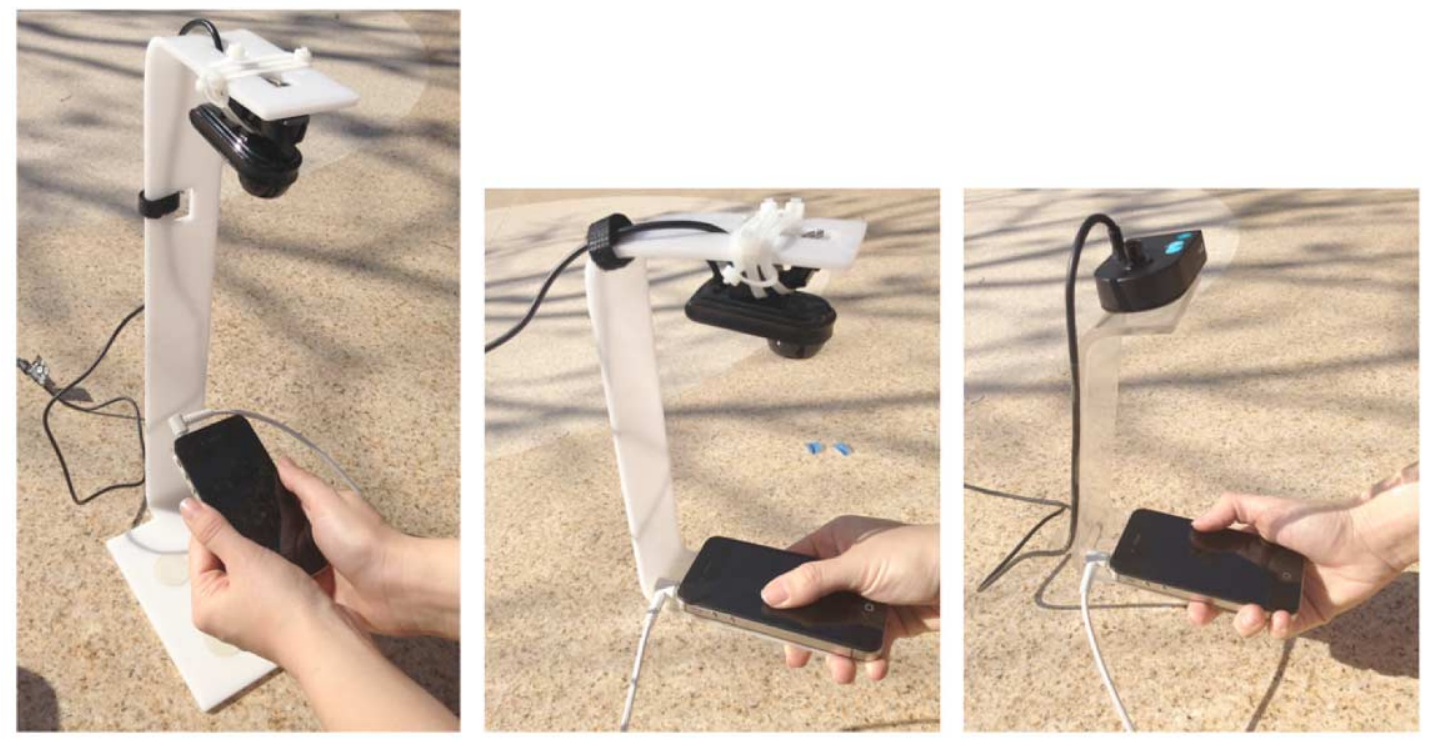

Figure 2. Our recording devices, from left: Overhead camera, white sled, clear sled

and a smartphone with attached headphones. The fourth station was the control station, which had a smartphone with headphones but no recording device.

Each participant sat at one of the four stations with an assigned researcher. The researcher moved his or her participant between stations according to a predetermined order.

Before playing either game, participants filled out a short survey asking how familiar they were with the two games, Ridiculous Fishing and Fruit Ninja. After the survey, the moderator described how to play each game and then allowed participants to play through each game twice in order to familiarize themselves with the games' rules and objectives. During this time, the mobile devices were not attached to the recording devices and researchers recorded the participants' baseline posture, handgrip, and body language. The smartphones had headphones attached for the entire session.

After participants tried each game, we attached the smartphones to the sled recording devices. For the stationary overhead camera, we told participants to hold the smartphone under the camera and reminded them to keep their heads out of view of the recording device, as well as keep the smartphone as flat as possible in relation to the camera. For the sleds, we handed the sled to each participant and encouraged them to hold the devices as naturally as possible. We did not force participants to hold the sleds if they set the sled down on the table while playing.

Participants played five trials of Ridiculous Fishing followed by five trials of Fruit Ninja at each station. After each trial, we recorded our assigned participants' scores. While the participants were playing, we noted qualitative observations with a focus on posture, handgrip, head position, and body language.

Every time the players completed all trials at a station, they completed a survey regarding their perceived performance with the recording device, how comfortable they felt with it, their ability to focus on gameplay at the station, and how aware of the recording device they were while playing the game. They rated each of these on a 5-point Likert scale. Researchers then moved with participants to the next station in their counterbalanced sequence and repeated this procedure for each of the remaining three stations.

When all participants had completed all four conditions, they completed one last survey question, which showed photos of each mobile recording device and asked participants to rank the devices in order of preference.

During each session, we stationed two to three researchers in the observation room who watched camera feeds of each station, noting any observation errors that occurred during the sessions. This included obstructed camera views and other obstacles to observation that resulted from the recording capture method.

\section{RESULTS}

To evaluate the results of the study, we used quantitative analysis of scores, player self-reported data, researcher notes, and notes from the observation room.

\section{Quantitative Analysis of Findings}

\section{Player Score}

To analyze the quantitative score results of this study, we used a series of mixed regression analyses with crossed and nested random effects. All models were run in R using the 
"Imer" function from the lme4 library [1]. The primary dependent measure of interest was the game score.

In the first model, we examined the overall effect of the recording devices across games. The scores for each game were Z-transformed separately across all users and all sessions so that the games had scores with equal mean and variance. The model included a fixed effects term for the station, dummy-coded with the control group as the reference group. Random effects were included for the session, for the individual participant within the session, for the order of the station within each participant (i.e., first station through four station, without reference to the device present at that station), and for the repetition of the game within each station (i.e., first through fifth replay of the game at that station). Finally, a random effect of the game was included, which was crossed with all other random effects because each participant completed the above structure for each of the two games.

In this model, there was a statistically significant omnibus effect of the station $(\mathrm{Chisq}(4)=11.2, \mathrm{p}=0.025)$, indicating that the different types of recording device did impact the scores of the players. Examining the individual stations, only the white sled showed a significant difference from the no-recording control station, with an estimated score decrease of -0.16 standard deviations versus control $(\mathrm{t}=$ $2.2, \mathrm{p}<0.05)$. The results are shown in Table 1.

In order to determine what was driving this effect, we performed several additional regression analyses. First, we repeated the above analysis separately for each game. Since there was no need to make the scores from each game commensurate, the dependent measure for each model was the untransformed game score. The random effect of game was removed from each model.

For Ridiculous Fishing, the results appeared similar to the overall results described above. The omnibus station effect was again significant $(\operatorname{Chisq}(4)=12.8, \mathrm{p}=0.012)$. The individual dummy-coded station effects were all negative in sign, indicating an overall decrease in score due to any kind of setup. However, the only station effect that appeared statistically significant was again the white sled, which penalized players by -22 points (from a baseline score of 86 points) $(\mathrm{t}=-3.4, \mathrm{p}<.01)$. Results are shown in Table 1 .

For Fruit Ninja, the results were quite different. First, the omnibus effect of the station, while present, was only marginally significant $(\operatorname{Chisq}(4)=7.85, \mathrm{p}=.097)$. Second, the pattern of results was different. The stations with overhead camera and white sled had effectively 0 impact relative to the no-recording control, while the effect of the clear sled was directionally positive. Results are shown in Table 1.

It seems the overall effects from the original analysis are primarily driven by the consistent effect on Ridiculous Fishing, but the impact on Fruit Ninja was more variable.

\begin{tabular}{|c|c|c|c|}
\hline \multicolumn{4}{|c|}{ Overall player score } \\
\hline Device & Estimate & Std. error & t value \\
\hline (Intercept) & 0.032456 & 0.143560 & 0.226 \\
\hline Overhead Cam & -0.069208 & 0.074315 & -0.931 \\
\hline White Sled & -0.162632 & 0.074315 & -2.188 \\
\hline Clear Sled & -0.000493 & 0.074315 & -0.007 \\
\hline Ridiculous Fishing player score \\
\hline Device & Estimate & Std. error & t value \\
\hline (Intercept) & 86.373 & 13.041 & 6.623 \\
\hline Overhead Cam & -7.493 & 6.573 & -1.140 \\
\hline White Sled & -22.220 & 6.573 & -3.380 \\
\hline Clear Sled & -10.267 & 6.573 & -1.562 \\
\hline \multicolumn{5}{|c|}{ Fruit Ninja player score } \\
\hline Device & Estimate & Std. error & t value \\
\hline (Intercept) & 269.83333 & 12.90359 & 20.911 \\
\hline Overhead Cam & -3.00667 & 11.38769 & -0.264 \\
\hline White Sled & 0.09333 & 11.38769 & 0.008 \\
\hline Clear Sled & 15.83333 & 11.38769 & 1.390 \\
\hline
\end{tabular}

Table 1. Player score analysis results

If we enter the game as a fixed instead of a random effect into our model, and allow it to interact with the station effect, then the omnibus Game*Station interaction term is marginally significant $(\mathrm{Chisq}(4)=7.9, \mathrm{p}=.095)$.

\section{Player Characteristics}

Having understood something about the overall impact of different types of recording devices, we were interested to know whether these results differ for different player demographics. User research is performed with a variety of groups of players including children, younger adults, and older adults. In this study, each of these groups was represented by separate sessions, with two sessions of five players for each type of player (children 8 to 12, adults 18 to 35 , and adults 40 to 60 ).

In order to evaluate the effect of station on each of these player types, we created planned orthogonal contrasts from the session identifier. The first component compared children to adults, taking the value -2 for children and the value 1 for both groups of adults. The second component compared younger adults to older adults, taking the value -1 for younger adults, 1 for older adults, and 0 for children. These predictors were then included as fixed effect terms in the across-game model and were allowed to interact with the station terms. 


\begin{tabular}{|c|c|c|c|c|}
\hline Ranking & 1st & 2nd & 3rd & 4th \\
\hline Control & $\mathbf{2 4}$ & 3 & 1 & 2 \\
\hline Overhead Cam & 1 & $\mathbf{1 3}$ & 9 & 7 \\
\hline White Sled & 2 & 1 & 10 & $\mathbf{1 7}$ \\
\hline Clear Sled & 2 & $\mathbf{1 3}$ & 11 & 4 \\
\hline
\end{tabular}

Table 2. Number of participants who selected each rank for each recording devices

The results revealed no systematic impact of player type on recording device impact. The omnibus effect of the Station*Age interaction was not statistically significant $(\operatorname{Chisq}(8)=6.0$, n.s. $)$, and no individual Station*Age term rose to the level of statistical significance (all ts $<1.1$, n.s.). It appears that the effect of different recording device, while possibly different for different types of games, does not differ systematically for users of different ages.

\section{Questionnaire Ratings}

The most objective measure of recording device impact on player performance is the final game score, as analyzed above. We can also measure the impact of the recording devices on players' perceptions of gameplay and ease of use, which may or may not correspond perfectly to any objective measure. Player ratings may be of particular interest when there are concerns about ergonomics or user comfort.

Players were asked to rank the stations in order of how well they felt they performed in each (rank $1=$ best, rank $4=$ worst). The results are shown in Table 2. Although we do not present a statistical significance test, the differences between the stations is clear. The majority of players chose the station with no recording device as leading to the best performance. The most frequent responses put the overhead camera or the clear sled in the second rank and the white sled in the lowest rank.

Players also answered a set of four questions about each of the four stations. Ranking questionnaires are useful for breaking ties between alternatives while ratings are useful for evaluating how players felt about each station individually. The four questions were:

1. Performance: "How do you feel you performed using this device?"

2. Comfort: "How comfortable or uncomfortable were you using this device?"

3. Focus: "How would you describe your ability to focus on the gameplay while using this device?"

4. Awareness: "How aware or unaware of this recording device were you while playing the game?"

The first three questions were asked of all four stations. The fourth question was asked about all stations other than the station with no recording device.

Among the results, the mean ratings (see Figure 3) again show the station with no recording device as the clear winner, and seem to favor the overhead camera and the clear sled over the white sled.

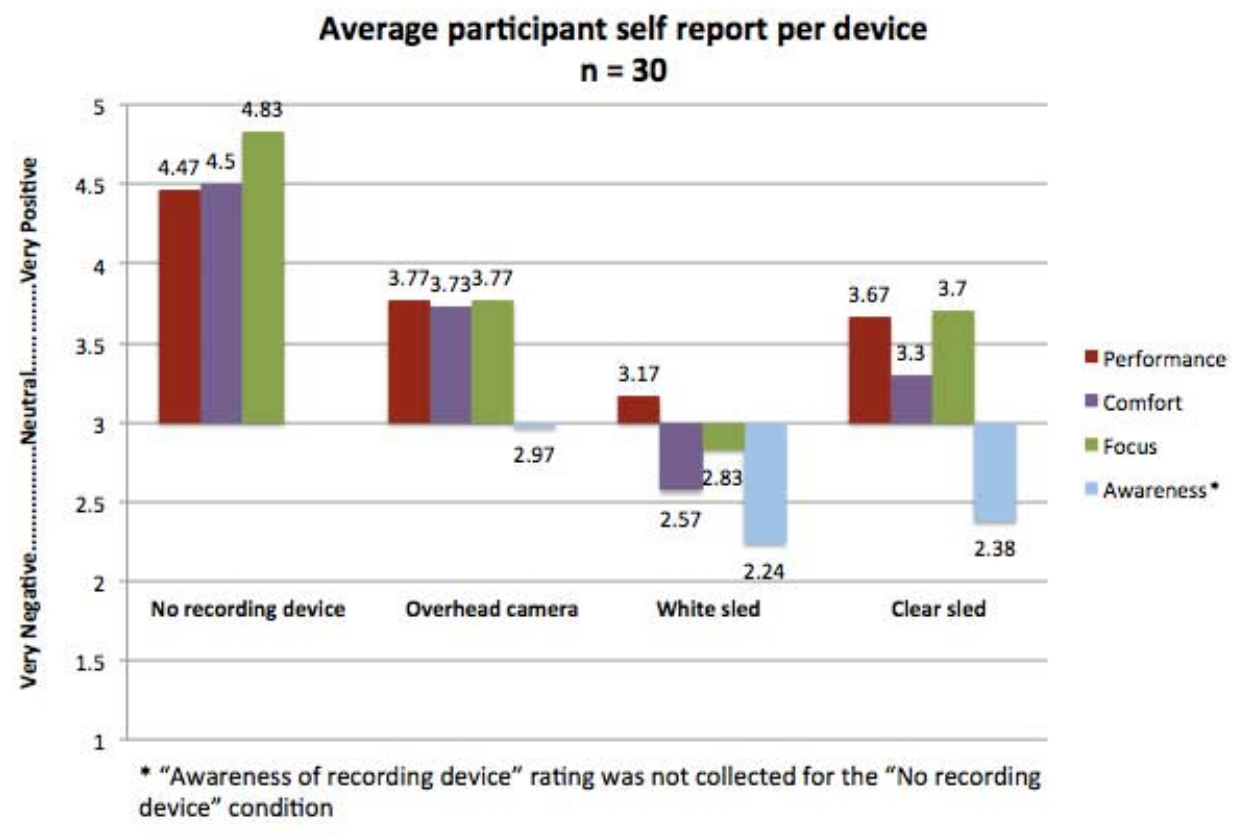

Figure 3. Participants rated each device in terms of performance, comfort, focus, and awareness 
The white sled was at the bottom of every measure. The average ratings are the simplest way to interpret these results since only the station with no recording device has an unambiguous distribution of responses.

The overall ranking preferences do not seem to be driven solely by any one aspect reflected in these questions. The station with no recording device is clearly preferred in all cases and the white sled always received the lowest average rating.

We wondered whether any of these questions help predict an individual's ranking of each station. Taking the ranking data and all ratings as numerical values on a continuous scale, we ran several more mixed regression models. Each question individually can help predict the overall rankings, with performance being the weakest predictor $(\operatorname{Chisq}(1)=13.9, \mathrm{p}<.0005)$ and the others all somewhat stronger $($ Chisq $(1)>20, p<.0001)$. Since all four questions are highly correlated with each other on an individual basis ( $r>0.5$ in all cases), no question adds significant value in the presence of the other three $(\mathrm{t}(1)<1.6, \mathrm{n} . \mathrm{s}$. $)$.

It seems that we can take any one or two of these questions as a valid gauge of a player's perception of their overall performance with a piece of user-testing equipment. Of the four questions, the focus rating appears to provide the most direct reflection of the overall rankings, but even the performance rating does a reasonable job. We believe the question of ability to focus could be a decent indicator of recording device impact.

\section{Participant Behavior and Observation Side Errors}

Qualitative comparison of adults and children during the study revealed that adults displayed more visible symptoms of fatigue and loss of enthusiasm when playing mobile games for $40+$ minutes. Adults were able to use the different recording devices more easily than the children, though this may be related to the physical limitations of the players. Children had difficulty adjusting their chairs to table height and their feet often did not touch the ground.

While players frequently cited the overhead camera as their preferred condition due to added freedom, this condition was suboptimal for observation. Observers were often unable to view the device based on the orientation of the iPhone, as players tilted the phone to view it better or pulled their hands/device out from under the camera. Added vertical freedom also required constant autofocusing of the camera, resulting in more observation errors. With this camera setup, children tended to lean in beneath the camera and put their heads in between the lens and the phone screen. This did not happen with adults except in a few cases where they were wearing hats that extended under the camera. Researchers frequently had to stop participants' gameplay in order to correct these observation issues.

While the two sleds had the best overall observer experience, including the ability to consistently see the gameplay and players' hands, several issues were common to both. The sleds' added weight changed the way players held and manipulated the mobile devices. Some players held the necks of the sleds as if they were handles.

The white sled caused unique player behavior errors. Players frequently complained that the sled was top heavy. Children dropped or lost control of the device due to its uneven weight distribution. These issues were more evident during Ridiculous Fishing because the use of the accelerometer required tilting the device back and forth. The plastic neck of the sled and the camera were in the way, which caused players to look at the mobile device from the side. Playing Fruit Ninja in landscape mode was difficult due to a lack of surface area between the iPhone and the sled base, which often resulted in the iPhone falling off the sled.

While many players stated a preference for the clear sled, observation errors still occurred in this condition. When players chose to play with the sled flat on the desk, they sometimes found that the device would slip away or fall over during play. Adding the crossbar to this sled for landscape play added weight to the device, which may have made participants more likely to put it down.

\section{CONCLUSION}

Performing usability research on mobile applications is important in helping development teams make successful products. Teams that can observe users interacting with their applications come away with an understanding of issues and behavior that assists them with making informed decisions. The scope of possible issues with mobile applications encompasses visual, gestural, and interaction challenges, so it is important for development teams to be able to observe both software and hardware interactions. In order for researchers and teams to observe all types of usability errors, it's crucial for the user to behave as naturally as possible, holding the device and interacting with the application as they normally would.

We set up this experiment to evaluate a variety of mobile recording devices and determine our current best solution for recording and streaming mobile user tests. All methods of recording caused some type of effect to player behavior, observed qualitative changes and, in the case of the white sled, significant changes in score. In addition, two of the four conditions provided poor or no observation of the tests: no recording and limited observation for the control and consistent problems with observing from the overhead camera. The two sleds provided good to great observation of players' screens and hands, but they caused some unnatural player behavior.

After analyzing the results of this study, we were able to determine which device offered the best observational data (hands and screen) while impacting players' natural behavior the least. While the overhead camera seems to cause fewer changes to player behavior than the sleds, the 


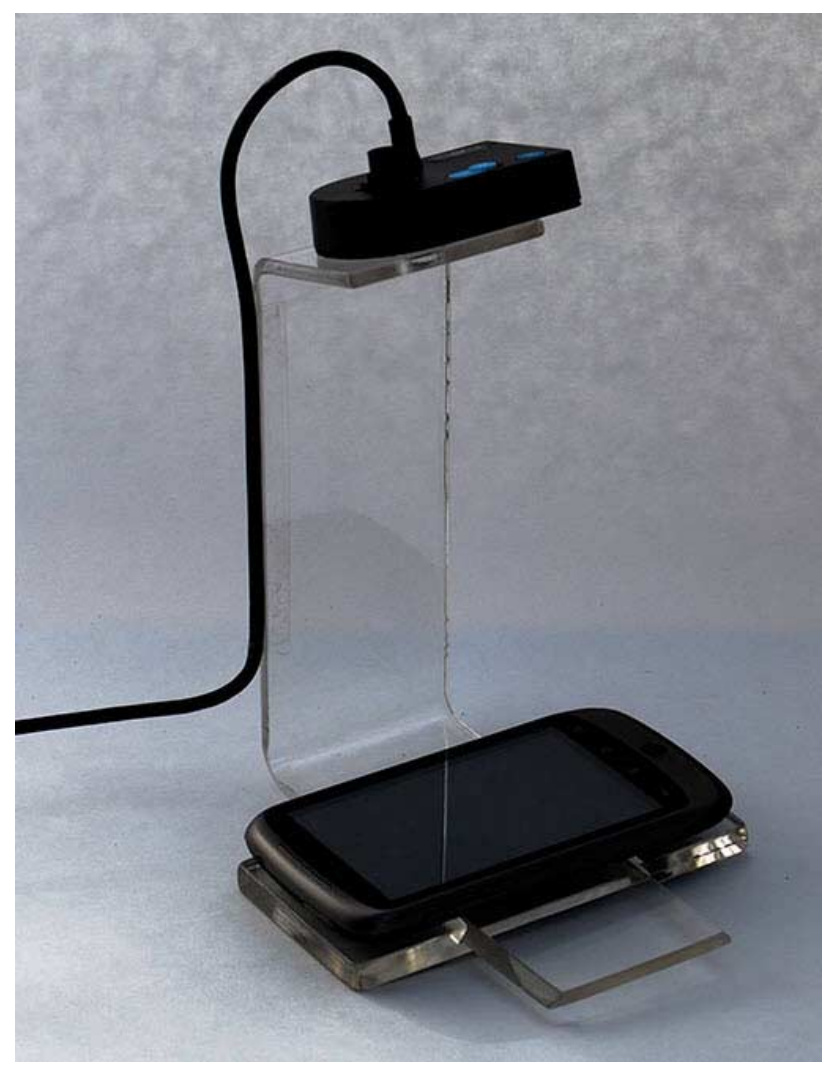

Figure 4. Based on the results of this study, the clear sled is our current best solution [7]

observational issues it causes means that the moderator must frequently intervene to correct the issue. With the two sleds offering the best observational data, it came down to a choice between the white sled and the clear sled. The white sled was consistently ranked the lowest in all metrics by players and was the only device that had a statistically significant negative effect on the player score. The white sled is larger and has poorer balance than the clear sled. These factors led to more severe and more frequent behavioral changes than we saw with the clear sled.

The clear sled has multiple positive attributes and its physical characteristics helped it perform best. It didn't cause statistical score change. It provided the best observational experience with clearly recorded hand position, fingers, and screen. The sled is clear, making it less noticeable to players and observers. The camera is small, light and flat, which allows it to be attached firmly. Without the landscape attachment, it's lighter than the white sled. With the attachment, it's only slightly heavier while adding physical support for a landscape-oriented device that is lacking from the white sled. It is compact, so the camera doesn't get in the way of the player's vision. Most importantly, the weight is well balanced. This makes it easier for players to hold for an extended period and assists when engaging with gesture heavy applications and applications using the accelerometer.

We have decided that the clear sled is our current best solution for our mobile research needs, both providing good viewing for the observers and not causing significant behavioral changes in players. However, we will continue to evaluate and iterate on our mobile recording methods.

\section{ACKNOWLEDGMENTS}

We would like to thank Liz Heron, Nathan Cook, Derek Speir, and Joseph Quevedo for their contributions in data collection, review, and moral support.

*Brooke White, Jeremy Glick, and Jason Yow are no longer employed by The Walt Disney Company.

\section{REFERENCES}

1. Bates, D., Maechler, M., Bolker, B., and Walker, S. Ime4: Linear mixed-effects models using Eigen and s4. R package version 0.999375-42. http://cran.rproject.org/web/packages/lme4/index.html.

2. Betiol, A.H. and de Abreu Cybis, W. Usability testing of mobile devices: A comparison of three approaches. Human-Computer Interaction-INTERACT, Springer Berlin Heidelberg (2005). 470-481.

3. Burghardt, D. and Wirth, K. Comparison of Evaluation Methods for Field-Based Usability Studies of Mobile Map Applications. International Cartographic Conference 2011 (2011).

4. Griffin, K., Kim, J., and Vakhariya, P. Overcoming Mobile Usability Testing Hurdles: A Mobile Payments Case Study. Proc. UPA 2007 (2007), 1-8.

5. Oulasvirta, A., and Nyyssönen, T. (2009). Flexible hardware configurations for studying mobile usability. Journal of Usability Studies 4,2 (2009), 93-105.

6. Ponoko iPhone usability testing sled. http://www.ponoko.com/design-yourown/products/iphone-usablilty-testing-sleds-6854

7. Poole, D. Smart Sled. http://smartsled.com.

8. Racadio, R., Rose, E., and Boyd, S. Designing and evaluating the mobile experience through iterative field studies. Proc. SIGDOC 2012, ACM Press (2012), 191196.

9. Schusteritsch, R., Wei, C. Y., and LaRosa, M. Towards the perfect infrastructure for usability testing on mobile devices. Ext. Abstracts CHI 2007, ACM Press (2007), 1839-1844.

10. Wei, C. Y. Capturing mobile phone usage: Research methods for mobile studies. Proc. IPCC 2007, IEEE (2007), 1-6. 